\title{
Hybrid Behavioral Control Architecture for the Cooperation of Minimalist Mobile Robots
}

\author{
Lounis ADOUANE and Nadine LE FORT-PIAT \\ Laboratoire d'Automatique de Besançon, UMR CNRS 6596 \\ 24 Rue Alain Savary, 25000 BESANCON, France \\ \{Lounis.Adouane \& Nadine.Piat\}@ens2m.fr
}

\begin{abstract}
This paper presents a hybrid control architecture based on subsumption and schemas motors principles in order to achieve complex and cooperative tasks. The control architecture implemented is constituted by a set of independent and elementary behaviors organized in layers of skills. Specific low-level behaviors, called altruistic behaviors and inspired by societies of insects (attractive or repulsive signals), are used to improve the efficiency of the control. Therefore, competitive and cooperative mechanisms are used in an unique hybrid architecture of control to perform a complex box-pushing task by a set of mini-robots. The analysis of an elevated number of simulations allows us to have statistical results (time to complete the task was chosen as performance criteria) which show the existence of an optimal number of robots to achieve the box-pushing task and underline the importance of the use of altruistic behaviors to enhance the cooperative task.
\end{abstract}

Index Terms - Cooperative robotics, Behavioral architecture of control, Altruistic behavior, Box-pushing task.

\section{INTRODUCTION}

Cooperative robotics constitutes an active research field especially for completion of complex tasks which requires coordination of several elementary entities. The behavioral architectures are based on the concept that a global behavior (complexe task) of a robot can be done by the coordination of several elementary behaviors. In the literature, two families of techniques of coordination exist, competitive and cooperative architectures.

In competitive architecture, the command is given by an unique behavior, which has been selected temporarily among a set of active behaviors. The principle of competition can be defined by a set of fixed priorities like in subsumption architecture where a hierarchy of the different behaviors is defined [1]. This principle can be also dynamic like in the actions selection architecture [2] in which the behavior with a great level of activation is selected, this level is recomputed all time.

In cooperative architectures, the final command is the result of a compromise or a fusion between controls proposed by different behaviors, which can be active at the same time. The schema-based architecture [3][4] uses in a general manner the same potential field technique [5][6] for the computation of the response of each elementary behavior and to encode the robot's behavioral response.
The box-pushing task "BPT" is among the reference task using by researchers who work in the field of cooperative robotics. Generally the reference task tests the efficiency and the robustness of their control strategy. In what follows we will mention some proposed control in the literature which deals with the BPT. Reference [7] propose different schemabased motor control systems, among others the one which is used to position correctly the robot around desired object to push. Reference [8] uses principally motivational behaviors, periodic communication between robots and the evaluation of a specific metric to perform the action selection strategy for the pushing of a long rectangular box. Reference [9] the authors shows the possibility of achieving a cooperative $\boldsymbol{B P T}$ without direct communication between the agents. In reference [10], the authors use eleven identical robots endowed with purely reactive behaviors, and specify transitions between behaviors using binary sensing predicates.

Our proposed architecture of control combine the two techniques of coordination mentioned above (i.e., the subsumption and motor schema principles) to obtain efficient control architecture to swarm robotics (i.e., with large number of robots) which roughly interact between them to realize the $\boldsymbol{B P T}$. We also introduce in this architecture of control the use of simple attractive and/or repulsive signals broadcasted by robots to increase their cooperation, and thus the execution of the $\boldsymbol{B P T}$.

Section II starts with the specifications of the box-pushing task and by the description of simulated mini-robots features. In section III, we present in details the proposed hybrid architecture of control. Section IV is devoted to the description and the analysis of the set of simulations. A brief description of MiRoCo simulator is also done. This paper ends with some conclusions and perspectives.

\section{BOX-PUSHING TASK SPECIFICATIONS}

Box-pushing task remains a privileged complex task for the study of the features of reactive and distributed control of a group of cooperative robots. Cooperative BPT is difficult to control [11], due to the multitudes of forces applied on the box (dry and/or viscous friction, directions and strength forces of contact applied by robots, weight...etc.). This task is made even more difficult due to the large dynamics of the entities in interaction (robots, obstacles, object to push, ...etc.) and 
by the use of robots that are very limited in structural (power delivered by the mini-robots), decisional and sensory point of views. Knowing the multiple difficulties generated by the task of pushing object, this one proves to be a very interesting task to show the multiple capabilities that an hybrid stimulusresponse control architecture can have on its realization.

The box-pushing task can be summarized as follows. We have a number $\boldsymbol{N}$ of mini-robots that must drive a box $\boldsymbol{B}$ towards a circular target $\boldsymbol{T}$. The displacement of $\boldsymbol{B}$ requires in less the cooperation of a critical number $\mathbf{N c}$ of mini-robots.

\section{A. ALICE mini-robot}

We adopted for the experimental platform, the general structure of the ALICE mini-robot (Fig. 1) built at the EPFL $^{1}$ [12]. ALICE mini-robot is based on a modular design, thus it is very easy to add and/or to take out new sensors and/or communication floors. The ALICE minirobot has very reduced dimensions $(2 \mathrm{~cm} \times 2 \mathrm{~cm} \times 2 \mathrm{~cm})$, and is equipped with a PIC16F877 microcontroller with only 8 Kwords Flash EPROM program memory capacity. That considerably reduces the size of the control program that can be implemented. ALICE maximum speed is of $4 \mathrm{~cm} / \mathrm{s}$ and it has four infrared sensors IS1, IS2, IS3, IS4 positioned respectively at $0^{\circ}, 45^{\circ}, 180^{\circ}$ and $-45^{\circ}$ in relation to the front face of the mini-robot. These sensors detect obstacles at a maximal distance of $4 \mathrm{~cm}$. This restricts the mini-robot to short localized information.

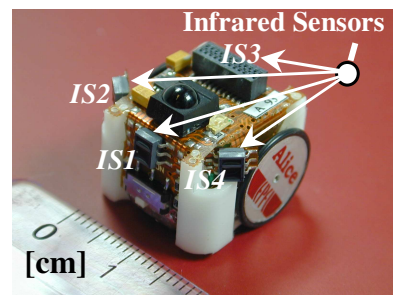

Fig. 1 ALICE mini-robot

\section{Control ARChitecture}

Because the mini-robots used in this work have very basic perceptual and decisional capabilities, we implement a purely stimuli-responses control inspired of individuals constituting the societies of insects [13].

We have defined a behavioral architecture, in which each behavior can be tested individually, in order to verify the reliability and the efficiency of this behavior to achieve a determined primitive. All these behaviors are gathered after in an unique and hybrid architecture (Fig. 2).

\section{A. General features of behaviors}

We adopt in what follows the next notations :

- command(Ori, Dis) : This response is given by the behaviors and consists of making the execution by the mini-robot of a rotation of "Ori" degrees and a

\footnotetext{
${ }^{1}$ Ecole Polytechnique Fédérale de Lausanne
}

translatory motion of "Dis" centimeters in a coupled movement. All behaviors, except for the behavior of Broadcast of the altruistic signals, have the possibility of sending the following discrete commands :

- Go Forward GF = command $\left(0^{\circ}, 1\right.$ cm),

- Go Backward GB = command $\left(0^{\circ}, \mathbf{- 1} \mathbf{~ c m}\right)$,

- Go Right-Rotate GRR = command $\left(-15^{\circ}, 0 \mathrm{~cm}\right)$,

- Go Left-Rotate GLR= command $\left(15^{\circ}, \mathbf{0} \mathbf{~ c m}\right)$,

- Go Right-Turn GRT = command $\left(-15^{\circ}, 1 \mathrm{~cm}\right)$,

- Go Left-Turn GLT $=\operatorname{command}\left(15^{\circ}, 1 \mathrm{~cm}\right)$,

- Remain There RT = command $\left(0^{\circ}, \mathbf{0} \mathbf{c m}\right)$.

The activation of Alignment, Box-pushing or Repositioning behaviors is linked to the direct contact between the mini-robot and the box to push.

We consider that at each moment the mini-robot can measure the angle $\theta$ that it makes with an active landmark in the environment. $\theta$ is between $-180^{\circ}$ and $180^{\circ}$.

\section{B. Description of the control architecture}

In the next sections, we start to present individually all elementary behaviors used in the proposed hybrid architecture of control. We will explain after the principles of coordination implemented between behaviors.

\section{1) Elementary behaviors:}

a) Exploration behavior: This behavior sends commands to the motors according to predetermined constant coefficients of the occurrence probability of each command.

b) Obstacles avoiding behavior: This behavior uses the infrared sensors only in all or nothing mode (boolean). It consists therefore in avoiding the obstacles according to a pre-established strategy giving the response according to the sixteen possible stimuli of the infrared sensors.

c) Attraction to the Box behavior: This behavior consists in attracting the mini-robot towards the box, which broadcasts light. This is performed using the mini-robots infrared sensors just in reception mode. The commands sent to the motors depend then on the values read on the four infrared sensors.

d) Alignment behavior: This behavior consists of insuring that the mini-robot is aligned with $\boldsymbol{B}$ before activating the behaviors of Box-pushing or the one of Repositioning. The idea is to control the relative angle that the mini-robot makes with $\boldsymbol{B}$, to be inside the interval $\left[-\eta^{\circ}, \eta^{\circ}\right]$.

e) Box-pushing behavior: After the detection of angles that the mini-robot makes with the box $\boldsymbol{B}$ and the one with the target $\boldsymbol{T}$, respectively $\theta_{1}$ and $\theta_{2}$ (Fig. 3), this behavior 


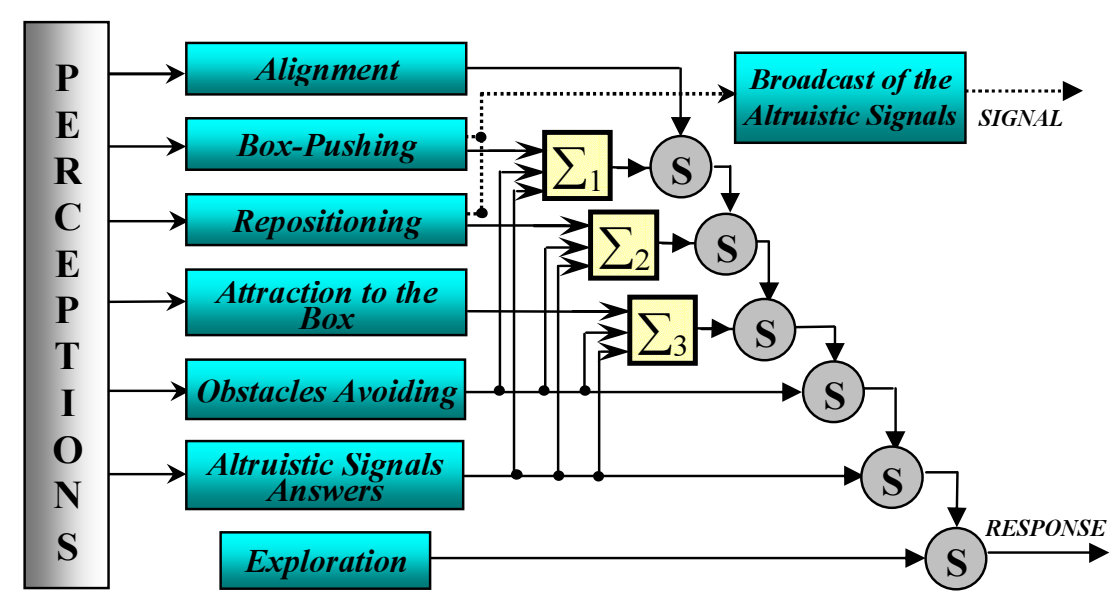

Fig. 2 Control based hybrid architecture

generates one response according to this following rule :

If $\left(\left(\left|\theta_{1}\right|\right.\right.$ and $\left.\left.\left|\theta_{2}\right|\right) \leq \theta_{\text {Max }}\right)$ Then apply the GF command Else apply RT command.

With : $\theta_{\text {Max }}$ is a positive constant less than $90^{\circ}$.

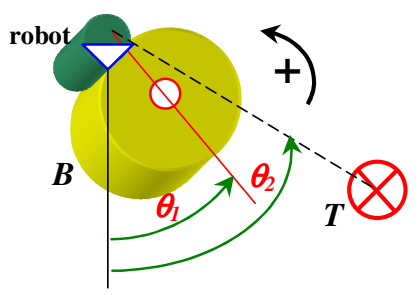

Fig. 3 Necessary information for performing the Box-pushing behavior

f) Repositioning behavior: The aim of this behavior is to appropriately, and quickly reposition the mini-robot around $\boldsymbol{B}$. According to the measured $\theta$ angle between the mini-robot and the target $\boldsymbol{T}$ at the instant $\mathrm{t}$, this behavior is going to generate a sequence of commands [14] like following :

- the mini-robot rotates with an angle $\alpha$ [degrees] as in

$$
\alpha=\boldsymbol{f}(\theta) .
$$

- after the rotation is ended, the mini-robot moves forward for a distance of $\boldsymbol{d}$ [centimeters] as in

$$
\boldsymbol{d}=\boldsymbol{g}(\theta)
$$

Where :

- $\boldsymbol{f}$ and $\boldsymbol{g}$ are linear functions of the $\theta$ angle,

- $\alpha$ and $\boldsymbol{d}$ are as big as $|\theta|$ is big,

- the direction of the rotation depends on the sign of $\theta$ as follows :

$$
\begin{aligned}
& \text { If }(\theta \leq 0) \text { Then apply GLR command } \\
& \text { Else apply GRR command. }
\end{aligned}
$$

g) Altruistic behaviors: The altruism is the fact to generate an effect (an action) with the objective to help its neighbours, and this without immediate obvious gain for the entity that generates this effect. The notion of altruism introduced in the proposed hybrid architecture of control, can be summarised by these two behaviors : the first one is the Broadcast of the altruistic signals and the second is the Altruistic signals answers. The first generates altruistic signals and the second exploits the altruistic signals generated by other robotics entities.

Broadcast of the altruistic signals behavior

This behavior is activated when behaviors of Box-pushing or Repositioning are activated (Fig. 2). This purely altruistic behavior consists in giving out attractive or repulsive signals (Fig. 4) as follows.

If $\left(|\theta|<\theta_{\text {Max }}\right)$ Then the given out signal is attractive

Else the signal is repulsive.

These signals have the objectives to attract mini-robots around the privileged zone (zone for which the mini-robots are susceptible to appropriately push $\boldsymbol{B}$ towards $\boldsymbol{T}$ ) and at opposite, to repulse mini-robots from non-privileged zone. Fig. 4 , shows roughly these two zones.

The range $\boldsymbol{E}$ and the intensity $\boldsymbol{I}$ of the signal are modulated according to the $\theta$ angle that the mini-robot makes with the target $\boldsymbol{T}$. This modulation is as large as :

- $|\theta|$ tends to $0^{\circ}$ in the case of attractive signal, indicating by this fact that the mini-robot is in the ideal position to push $\boldsymbol{B}$.

- $|\theta|$ tends to $180^{\circ}$ in the case of repulsive signal, indicating by this fact that mini-robot is positioned at opposite to where it must be to push $\boldsymbol{B}$ towards $\boldsymbol{T}$.

\section{Altruistic signals answers behavior}

The answers to the altruistic signals are commands that attract the mini-robot towards the most attractive altruistic signals (of bigger intensity) and move away from the most repulsive altruistic signals read by the four infrared sensors. 


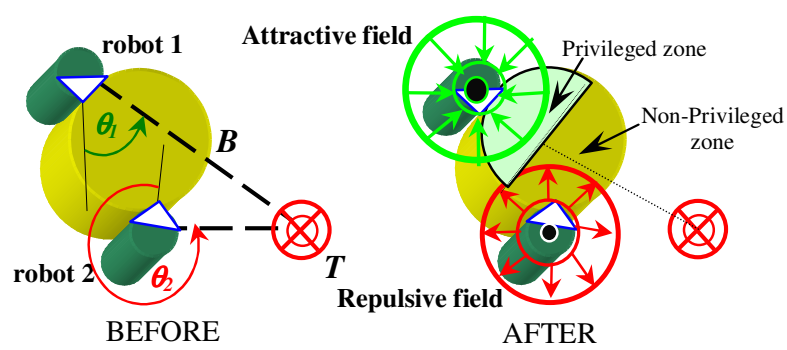

Fig. 4 Attractive and repulsive signals

2) Coordination of behaviors: We now explain the coordination defined between the different behaviors.

a) Subsumption coordination: Classically in subsumption architecture, behaviors of high-level can inhibit at any moment the responses generated by low-level behaviors.

This kind of hierarchy is managed entirely in our architecture of control via the responses (commands) generated by the behaviors as follows.

In addition to all the possible responses given by the behaviors for corresponding stimuli of sensors, we add for each behavior a specific response called Refuge Response "RR" [14] generated for a particular stimulus of sensors. We notice that :

- a distinct $\boldsymbol{R} \boldsymbol{R}$ is assigned to each elementary behavior,

- each behavior knows the $\boldsymbol{R} \boldsymbol{R}$ corresponding to the behavior which is one level hierarchically superior.

These two points allow to a low-level behavior to activate its command instead the one of a high-level only when it detects the activation of the Refuge Response corresponding to this one (the high-level behavior). Thus by chain effect, we realize the hierarchical coordination.

b) Fusion organisation: The implementation of our control architecture based only on the previous subsumption coordination [14] leads us to observe the existence of some microscopic undesirable effects, which are directly linked to the elevated number of mini-robots in the immediate surrounding of the box to push. To avoid these undesirable effects (see section "Interest of using fusion blocks"), we introduce fusion blocks of behaviors $\left(\Sigma_{1}, \Sigma_{2}, \Sigma_{3}\right.$ (Fig. 2)), which compute a command according to the commands given by merged behaviors. The principle of this fusion is explained below :

For each command " $\left.\operatorname{command}_{i}\left(\boldsymbol{O r i}_{i}, \boldsymbol{D i s}_{i}\right)\right|_{i=1 . .3}$ " in input of a fusion block $\left.\Sigma_{j}\right|_{j=1 . .3}$ (Fig. 2), two parameters are associated :

i) a gain, respectively $\left.\boldsymbol{g}_{j i}\right|_{i=1 . .3}$ that represents the weight of the corresponding command $_{i}$ as :

$$
\begin{aligned}
& \sum_{i=1 . .3} \boldsymbol{g}_{j i}=1 \\
& \text { with } \left.:\left.\boldsymbol{g}_{j i}\right|_{i=1 . .3} \in\right] 01[
\end{aligned}
$$

ii) and a vector $\vec{v}_{j i}$ which have :

- as module the gain " $\mathbf{g}_{j i}$ "

- and as orientation "Ori ${ }_{i}$ "

The output of the fusion block $\left.\Sigma_{j}\right|_{j=1 . .3}$ is a command " $\operatorname{command}_{j}\left(\mathrm{Ori}_{j}, \mathrm{Dis}_{j}\right)$ " given by this method :

If Box-pushing, Repositioning, or Attraction to the box behaviors give their $\boldsymbol{R R}$. Then the corresponding fusion block (respectively $\Sigma_{1}, \Sigma_{2}$ or $\Sigma_{3}$ ) gives in output this $\boldsymbol{R} \boldsymbol{R}$ which permit to not subsume behaviors and/or fusions blocks of lower levels.

Else the command com $_{j}$ is calculated as follows :

i) "Ori ${ }_{j}$ " is the orientation of $\vec{V}_{j}$ vector as in

$$
\vec{V}_{j}=\sum_{i=1 . .3} \vec{v}_{j i} .
$$

ii) and "Dis ${ }_{j}$ " is defined as scalar summation as in

$$
\text { Dis }_{j}=\sum_{i=1 . .3}\left(\mathbf{g}_{j i} \cdot \text { Dis }_{i}\right) .
$$

c) Interest of using fusion blocks: The main motivations to use fusion blocks are to improve the pure subsumption architecture [14] and to avoid undesirable effects linked to the elevated number of mini-robots in interaction. The details of motivations are explained next :

For the fusion block " $\Sigma_{1}$ ", we have note that when minirobots push the box at the same time, their trajectories go inevitably intersect (Fig. 5). These effects occur because the mini-robots tend always to push towards the centre of the box. To avoid this, the idea is to remain active the Obstacles avoiding behavior during the activation of the Box-pushing behavior. This is done in order to maintain always a minimal distance between mini-robots. As for the contribution of altruistic signals (if it exists in the environment) to this fusion block, it consists to attract the mini-robots closer to the most suitable zone to push (i.e., where the angle $\theta$ of observation of the target and the box tends to zero). Because the minirobots which are closer to this point, broadcast more intense attractive signal which attract other mini-robots towards it.

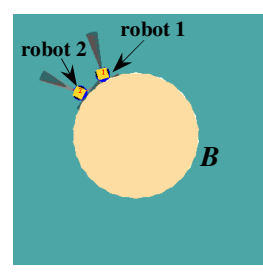

(a) Time $=\mathrm{T} 1$

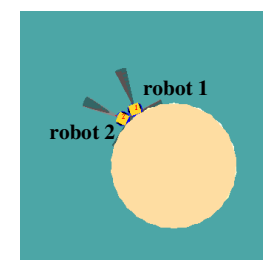

(b) Time $=\mathrm{T} 1+\triangle \mathrm{T}$
Fig. 5 Observed effect without using Obstacles avoiding behavior when mini-robots push the box

The fusion block " $\Sigma_{2}$ "makes so that the repositioning of mini-robot becomes more quickly towards the privileged zone (contribution of altruistic signals) while avoiding other mini-robots in the immediate surrounding of the box 
(contribution of Obstacles avoiding behavior).

The fusion block " $\Sigma_{3}$ " is linked directly to the behavior of Attraction to the box. The main contribution of the Obstacles avoiding behavior consists in preventing that the mini-robots would be one behind the other (Fig. 6(a)) which stops them thus to interact directly with the box.

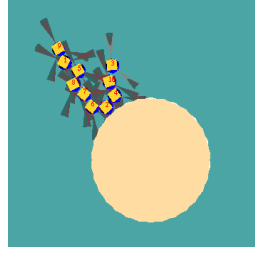
gain (a) With a very small

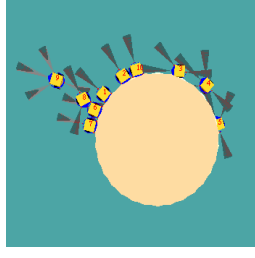

(b) With appropriate gain
Fig. 6 Influence of the gain of Obstacles avoiding behavior in the fusion block $\Sigma_{3}$

\section{Simulations}

To estimate the relevance of the proposed control architecture, and to observe the importance of altruistic signals on the realization of a cooperative $\boldsymbol{B P T}$ (where the mini-robots are always in very strong interaction), we developed a simulator called MiRoCo (Fig. 7) to realize a very large number of simulations (some thousands) in order to make statistical studies. The criteria chosen to verify the relevance of the control architecture, is the evolution of time of execution of the $\boldsymbol{B P T}$ according to :

- the number of mini-robots $\mathbf{N}$,

- the minimal number of robots to displace the box Nc,

- the use or not of altruistic signals.

\section{A. MiRoCo simulator}

MiRoCo (Mini-Robotics Collective) simulator (Fig. 7) developed in the $\mathrm{LAB}^{2}$ is dedicated in general to the cooperative mobile robotics. The $\mathrm{MFC}^{3}$ of Visual $\mathrm{C}++$ has been used as support to the implantation of MiRoCo. It is based therefore on oriented object architecture in which are defined separately all entities constituting the environment (robots, box to push, obstacles, target, model of displacement of the box...etc.). The interaction of all entities is very accurate, so it allows us therefore to have reliable simulations.

The display aspect is managed completely by using the OpenGL ${ }^{4}$ graphic library. This gives us a very good indications about the dynamic of interactions of all entities.

\section{B. Organisation of the sets of simulations}

We achieve two sets of simulations with the proposed control architecture (Fig. 2), those with altruistic signals and those without. For each set of simulations, we proceed as

\footnotetext{
${ }^{2}$ Laboratoire d'Automatique de Besançon

${ }^{3}$ Microsoft Foundation Class Library

${ }^{4}$ Open Graphics Library
}

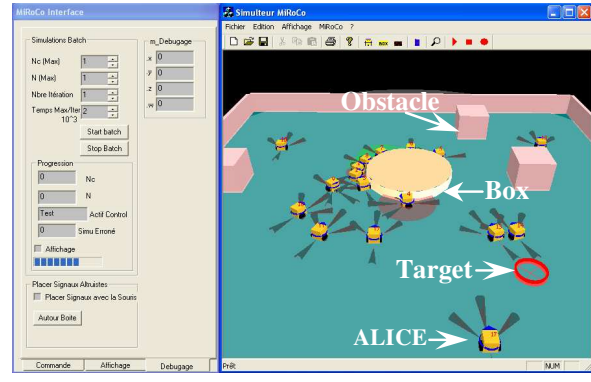

Fig. 7 MiRoCo simulator

follows : For a given number of $\mathbf{N c}$ (minimum number of mini-robots necessary to push the box) and $\mathbf{N}$ (number of mini-robots as $\mathbf{N} \geq \mathbf{N c}$ ), we made a number of simulations equal to Nbsim. The initial positions of mini-robots change randomly for every simulation in order to have variable initial conditions and also more reliable statistical results.

\section{Simulations Results}

Fig. 8(a), represents the simulations done with a control without altruistic behaviors and Fig. 8(b), those done with it. Each of the two 3D curves represent the evolution of the average time of execution of the box-pushing task according to the number of mini-robots $\mathbf{N}$ (with $\mathbf{N}$ going from $\mathbf{N c}$ to $(\mathbf{N c}+15)$ and this for $\mathbf{N c}=1 . .4)$. The number of iterations in each point represents the average of $\mathbf{N b s i m}=50$ simulations with the same $\mathbf{N c}$ and $\mathbf{N}$ but for different initial positions of the mini-robots. The maximum number of iterations to the execution of the simulations is fixed to 800 . We set in our simulations the following parameters : $\eta=15^{\circ}, \theta_{\mathbf{M a x}}=50^{\circ}$, $\boldsymbol{E}_{\text {Max }}=2 \mathrm{~cm}$, and

$$
\left\{\begin{array}{l}
\alpha=-\operatorname{sign}(\theta)\left(\frac{|\theta|}{20}+70\right) \\
\boldsymbol{d}=\frac{|\theta|}{45}
\end{array} .\right.
$$

With $\operatorname{sign}(\theta)$ is the representative function of the algebraic sign of $\theta$.

The gains of the fusion blocks are empirically obtained while following the expected behaviors of mini-robots considered in section "Interest of using fusion blocks".

$-\Sigma_{1}: g_{11}=0.50, g_{12}=0.40, g_{13}=0.10$

$-\Sigma_{2}: \boldsymbol{g}_{21}=0.50, \mathbf{g}_{22}=0.45, \mathbf{g}_{23}=0.05$

$-\Sigma_{3}: \mathbf{g}_{31}=0.20, \mathbf{g}_{32}=0.16, \mathbf{g}_{33}=0.64$

With : $\boldsymbol{g}_{11}, \boldsymbol{g}_{21}, \mathbf{g}_{31}$ are gains of commands corresponding respectively to Box-pushing, Repositioning and Attraction to the box behaviors, $\mathbf{g}_{12}, \mathbf{g}_{22}, \mathbf{g}_{32}$ correspond to Obstacles avoiding behavior and $\mathbf{g}_{13}, \mathbf{g}_{23}, \mathbf{g}_{33}$ correspond to Altruistic signals answers behavior.

These simulations allow us to observe following tendencies : Fig. 8(a) and Fig. 8(b), shows that the time of execution of $\boldsymbol{B P T}$ decreases when the number $\boldsymbol{N}$ of mini-robots increases, however this time increases when $N \boldsymbol{c}$ increases. We 
note also the existence of an optimal number $\mathbf{N}^{*}$ of minirobots to complete the $\boldsymbol{B P T}$, beyond it there is not (or very little) enhancement of the time of execution. We see also that Fig. 8(b) is smoother than Fig. 8(a). This shows that the use of low-level of communication (attractive and/or repulsive signals) give to the cooperative task more coordination, and robustness of interactions between mini-robots.

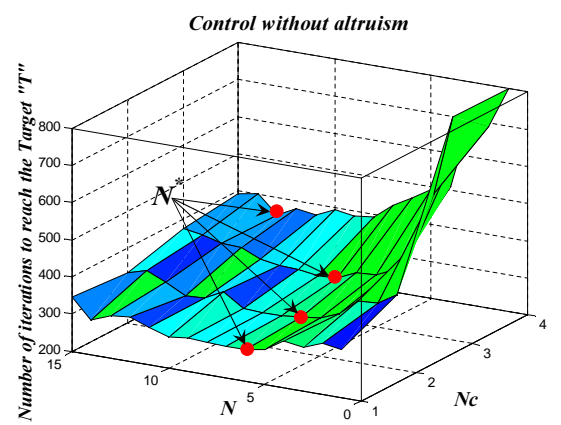

(a)

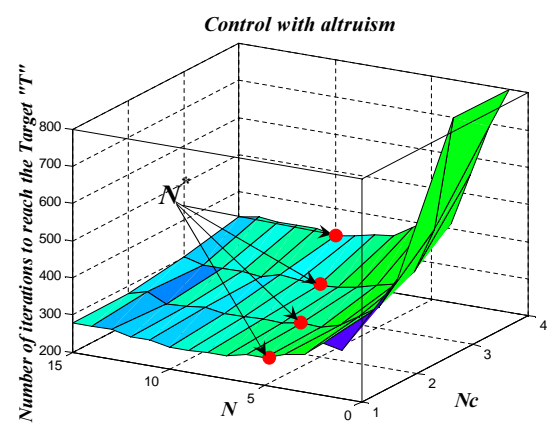

(b)

Fig. 8 Evolution of the number of iterations according to $\mathbf{N}$ and $\mathbf{N c}$

Fig. 9, represents the difference of numbers of iterations between control with altruistic behaviors and the one without for the execution of the BPT. The analysis of the 3D curve shows a very substantial gain, brought by the implicit cooperation through the altruistic signals (the middle time improvement for the realization of the $\boldsymbol{B P T}$ is about of $12.24 \%)$.

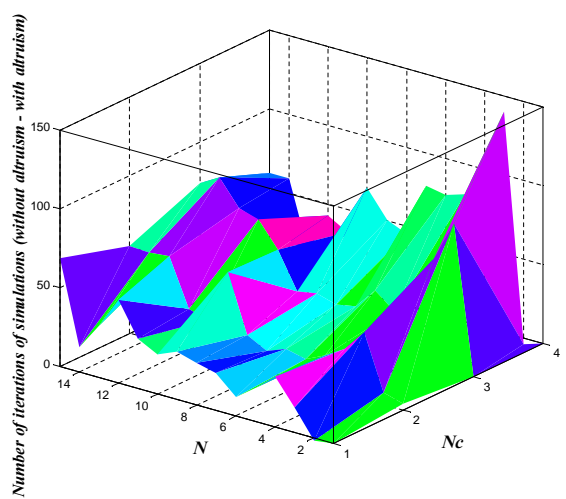

Fig. 9 Gain of time brought by altruistic signals

\section{CONClusions ANd Perspectives}

The proposed hybrid control architecture is very efficient to the realization of complex cooperative task like the one of the box-pushing which requires big interaction between a lot of mini-robots. We could note also the importance of the broadcasting of simple altruistic signals for the improvement of the $\boldsymbol{B P T}$ in term of time to complete the task.

To summarize, the proposed reactive control remains extremely interesting seeing its intuitive aspect, robustness and flexibility for the resolution of complex tasks as for the $\boldsymbol{B P T}$.

The future works are going firstly to implement the proposed hybrid architecture on the mini-robots ALICE, in order to test the efficiency of this hybrid control architecture in real situations. Secondly, knowing the multitude of parameters to be fixed, we will proceed by learning to determine the optimal parameters of control.

\section{REFERENCES}

[1] R. A. Brooks, "A robust layered control system for a mobile robot," IEEE Journal of Robotics and Automation, vol. RA-2, pp. 14-23, March 1986.

[2] P. Maes, "The dynamics of action selection," in Proceedings of the Eleventh International Joint Conference on Artificial Intelligence (IJCAI), Detroit, 1989, pp. 991-997.

[3] R. C. Arkin, "Motor schema-based mobile robot navigation," International Journal of Robotics Research, vol. 8, no. 4, pp. 92-112, 1989.

[4] T. Balch and R. C. Arkin, "Motor schema-based formation control for multiagent robot teams," in Proceedings of the First International Conference on Multiagent Systems (ICMAS'95). San Francisco, CA, USA : AAAI Press, 1995, pp. 10-16.

[5] O. Khatib, "Real-time obstacle avoidance for manipulators and mobile robots," The International Journal of Robotics Research, vol. 5, pp. 90-99, Spring 1986.

[6] R. C. Arkin, Behavior-Based Robotics. The MIT Press, 1998.

[7] R. Emery and T. R. Balch, "Behavior-based control of a non-holonomic robot in pushing tasks," in ICRA, Seoul-Korea, May 2001, pp. 23812388.

[8] L. E. Parker, "Adaptive heterogeneous multi-robot teams," in Neurocomputing, special issue of NEURAP '98: Neural Networks and Their Applications, vol. 28, 1999, pp. 75-92.

[9] S. Yamada and J. Saito, "Adaptive action selection without explicit communication for multirobot box-pushing," IEEE Transaction On Systems, Man And Cybernetics-Part C : Application And Reviews, vol. 31, No.3, pp. 398-404, August 2001.

[10] C. R. Kube and H. Zhang, "Task modeling in collective robotics," Autonomous Robots, vol. 4(1), pp. 53-72, 1997.

[11] K. M. Lynch, "The mechanics of fine manipulation by pushing," in Proceeding of the IEEE International Conference on Robotics and Automation, Nice-France, May 1992, pp. 2269-2276.

[12] G. Caprari, T. Estier, and R. Siegwart, "Fascination of down scaling alice the sugar cube robot," Journal of Micro-Mechatronics, vol. VSPUtrecht, pp. 177-189, 2002.

[13] E. Bonabeau, M. Dorigo, and G. Theraulaz, Swarm intelligence 'from Natural to Artificial systems'. Oxford University Press, 1999.

[14] L. Adouane and N. L. Fort-Piat, "Bio-inspired behaviours of a group of microrobots for cooperative box-pushing task," Saitama-Japan, sept. 2003, pp. 483-488. 